\title{
Characterising movement patterns of medaka (Oryzias latipes) in response to copper analysed by using a self-organising map
}

\author{
C.-W. Ji ${ }^{1} \&$ Y.-S. Park ${ }^{2}$ \\ ${ }^{1}$ Department of Biological Sciences, Pusan National University, \\ Republic of Korea \\ ${ }^{2}$ Department of Biology and The Korea Institute of Ornithology, \\ Kyung Hee University, Republic of Korea
}

\begin{abstract}
The automatic detection of animal movement behaviours in response to disturbances is considered an efficient tool for the bio-monitoring of ecosystems. Behaviours, however, can be difficult to analyse, due to complexity of the data. In this study, we characterised the movement patterns of medaka (Oryzias latipes) in response to low concentrations $(1 \mathrm{mg} / \mathrm{L})$ of copper ion $\left(\mathrm{Cu}^{2+}\right)$ using a self-organising map (SOM) with movement parameters. Test specimens were observed individually in an aquarium $(40 \mathrm{~cm} \times 20 \mathrm{~cm} \times 10 \mathrm{~cm})$, and their position was detected from the side at 0.25 -sec intervals using a television camera. Movement parameters such as speed, angle, and distance were calculated for 3 different segment lengths, with 3,6 , and 12 points indicating the duration of observation of $0.5 \mathrm{sec}, 1.25 \mathrm{sec}$, and $2.75 \mathrm{sec}$, respectively. The effects of copper treatment on movement patterns of medaka were evaluated with different segment lengths, and the discrimination efficiency was compared. Our results showed that the movement activity after $\mathrm{Cu}^{2+}$ treatment decreased, and changes in movement tracks caused by $\mathrm{Cu}^{2+}$ treatment were identified with segmentation using SOM. These computational approaches could be used for in situ bio-monitoring and early warning systems.

Keywords: movement pattern, response behaviour, copper, chemical treatment, medaka, early warning system, on-line monitoring, segment size.
\end{abstract}




\section{Introduction}

Biological systems are composed of hierarchical structures, ranging from the small-scale structures of molecules and cells to the larger scale structures of tissues, organs, organ systems, organisms, populations, and communities. Each of these responds in different ways to environmental changes. To evaluate biological responses at the molecular or cellular level, analytical methods, toxicological assays, and dose mortality assays are used. Responses at these small-scale levels are rapid, and the analytical methods provide accurate information (Figure 1). However, the information provided by such studies is usually limited to the local level, and fails to provide a comprehensive representation of the impacted ecosystems on a large scale [1, 2]. For large-scale biological systems, assessments of biodiversity and ecosystem function are used to evaluate the impacts of disturbances on the target ecosystems. On such a large scale, responses are slow and often imprecise, but their ecological relevance is high due to the accumulating effects of disturbances or stresses on the system.

\begin{tabular}{|c|c|c|}
\hline Ecosystem & \multirow{3}{*}{$\begin{array}{l}\text { Assessment of } \\
\text { biodiversity \& } \\
\text { ecosystem } \\
\text { function }\end{array}$} & \multirow{3}{*}{$\begin{array}{l}\text { - provide an overall idea of disturbance } \\
\text { impact on ecosystems. } \\
\text { - do not deliver detailed and precise } \\
\text { information }\end{array}$} \\
\hline Community & & \\
\hline Population & & \\
\hline Organism & \multirow{2}{*}{$\begin{array}{l}\text { 'integrative' } \\
\text { assessment }\end{array}$} & \multirow{3}{*}{$\begin{array}{l}\text { - Behavioral monitoring of organisms could } \\
\text { efficiently fill the gap between the large- } \\
\text { scale field surveys and the small-scale } \\
\text { chemical tests. }\end{array}$} \\
\hline Organ system & & \\
\hline Organ & & \\
\hline Tissue & & \\
\hline Cell & \multirow{2}{*}{$\begin{array}{l}\text { Analytical methods: } \\
\text { Chemical/toxicologic } \\
\text { al assays, dose- } \\
\text { mortality assays }\end{array}$} & \multirow{3}{*}{$\begin{array}{l}\text { - provide more accurate data } \\
\text { - information obtained is usually limited to } \\
\text { the local level and fails to address a } \\
\text { comprehensive representation on the } \\
\text { affected ecosystems. }\end{array}$} \\
\hline Molecule & & \\
\hline & & \\
\hline
\end{tabular}

Figure 1: Schematic diagram of biological assessments of disturbances at different biological scales.

Therefore, methods to link small- and large-scales studies, to achieve an integrative assessment of biological systems, have been developed $[1,3,4]$. Animal behavioural monitoring is effective for filling the gap between largescale and the small-scale assessments. Animal movement behaviour can be observed continuously.

For effective ecosystem management, it is useful to establish early warning systems for ecosystem disturbances. Accordingly, the automatic detection of animal response behaviours to disturbances has received considerable attention as an early warning system tool in aquatic ecosystems. Various methods have been proposed: circling paths of gametes or the meander searches by isopods [5], a complex directional autocorrelation function with monotonic decay in 
interpreting movement behaviour [6], fractal dimensions of movement pathways [7-10], movement velocity [4], two-dimensional fast Fourier transform [1], hidden Markov models [11, 12], permutation entropies [13], and Shannon entropy [2].

Although various behavioural monitoring systems have been developed, there are still many improvements necessary. Continuous behavioural monitoring is complex, because of the large number of data points provided by a continuously recorded time series. The behavioural data for movement tracks of animals are highly non-linear and variable, making it difficult to analyse data and extract useful information.

Previous studies have shown that movement parameters such as distance, acceleration, and speed are efficient for monitoring changes of movement behaviours of test animals responding to disturbances [1,3]. Although these computational methods extract useful information, they require high computational cost and their parameters are highly condensed. Therefore, it is necessary to develop relatively simple methods for detecting behaviours of test animals in response to environmental disturbances. Furthermore, it is unclear how many segments in the movement tracks are necessary to efficiently detect the responses of animals to disturbance. A large number of segments involve high computational cost, and result in complex interactions between parameters. In this study, we aimed to 1) determine the movement behaviour of animals responding to a toxic substance, and 2) evaluate the effects of varying numbers of segments in the movement tracks on the detection efficiency after exposure to a toxic substance.

\section{Methods}

\subsection{Test specimens and movement observation}

Medaka (Oryzias latipes) was used as the test specimen. This species is common and relatively easy to observe, and its general biological information is readily available. Medaka stock populations, obtained from the Korea Research Institute of Chemical Technology (KRICT), were maintained in a glass tank and were fed an artificial dry diet $\left(\right.$ Tetramin $^{\circledR}$ ) under the light regime of L10:D14 (lights on, 10:00; lights off, 20:00) at a water temperature of $23 \pm 1{ }^{\circ} \mathrm{C}$ [2]. Tap water, dechlorinated by adding $\mathrm{Na}_{2} \mathrm{~S}_{2} \mathrm{O}_{3}(30 \mathrm{mg} / \mathrm{L})$ and by providing air under sunlight for 2 or 3 days, was used [14]. Test organisms (aged 6-12 months) were randomly chosen, and were placed individually in a $10-\mathrm{L}$ glass aquarium $(40 \times$ $20 \times 10 \mathrm{~cm})$. Copper $(\mathrm{Cu})$ at a concentration of $1 \mathrm{mg} / \mathrm{L}$ was added to the aquarium. The $\mathrm{LC}_{50}$ was $5 \mathrm{mg} / \mathrm{L}[2,15]$. Twenty individuals were continuously observed from side view for 4 days: for 2 days prior to treatment and subsequently for 2 days after treatment [2].

Medakas were continuously observed using the automated behaviour observation system, which consisted of an observation aquarium, a CCD camera, and a computer with an $\mathrm{A} / \mathrm{D}$ converter and an image recognition system (Figure 2). The test organisms were scanned continuously with the CCD camera, 
and the scanned analogue images were converted to digital images using a video overlay board every $0.25 \mathrm{sec}$. The digital images were processed by the image recognition system to identify the location of the test specimen every $0.25 \mathrm{sec}$ [2]. The observation system, including the software for digital image processing and other mathematical analyses, was developed by the authors.

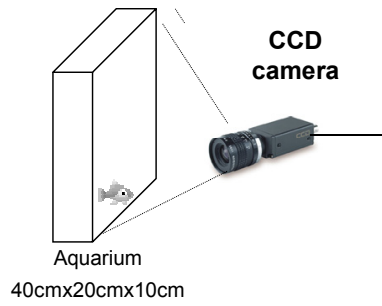

Observation aquarium

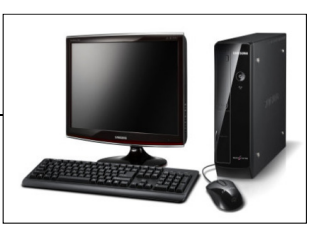

Personal computer

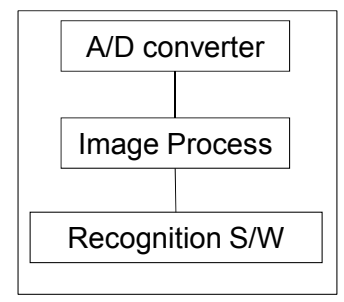

Software

Figure 2: Observation system for automatically recording and analysing movement behaviour.

\subsection{Data analyses}

Based on the movement tracks of the test specimens, movement parameters (e.g. speed) were selected for comparing the movement activity before and after copper treatment. A paired $t$-test was used to test the differences before and after treatment. Based on our experience of test specimens and the results from previous studies of continuous observation of response behaviours $[1,3,16]$, the parameters of speed $(\mathrm{mm} / \mathrm{sec})$, angle $(\mathrm{rad} / \mathrm{sec})$, angle change per movement distance, and movement distance $(\mathrm{mm})$ between segment points were measured for characterizing movement of the segments (Figure 3). Parameters were calculated at different movement lengths (number of segments); duration of movement was presented as 3, 6, or 9-point segments indicating $0.5 \mathrm{sec}, 1.25$

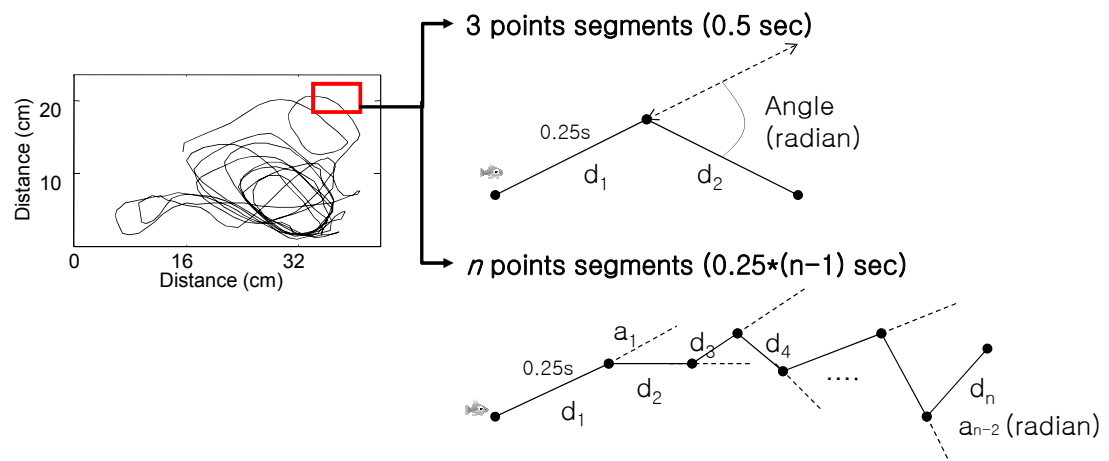

Figure 3: Segmentation of movement tracks. 
sec, and $2.0 \mathrm{sec}$, respectively (Figure 3 ). The movement distance was calculated each $0.25 \mathrm{sec}$; therefore, $n$ numbers of movement distance parameters were obtained for $n$ point segments tracks.

Average values and statistical analyses were used for quantifying the differences before and after treatment. Subsequently, the movement patterns were evaluated based on the behavioural parameters of a Self-Organizing Map (SOM; [17]), an unsupervised artificial neural network. The SOM, consisting of 2 layers of input and output, performs a non-linear projection of the data in low dimension [17], and provides a comprehensive representation of complex data to allow patterning. Each layer consists of neurons with inter-connecting weight vectors. In the learning process, the Euclidian distances between weight vector $\boldsymbol{w}$ and input vector $\boldsymbol{x}$ are calculated. The output layer consists of $D(150=15 \times 10$ in this study) output neurons on a two-dimensional hexagonal grid. Among $D$ neurons, the best matching neuron (that with the minimum distance) is chosen. The new weight vectors are updated for the best matching neuron and its neighbours according to the following equation. The weight vectors of other neurons are not changed.

$$
w_{i}(t+1)=w_{i}(t)+\alpha(t)\left[x(t)-w_{i}(t)\right]
$$

where $t$ is the iteration time, and $\alpha(t)$ is a learning rate factor. This process amounts to training the network to classify the input vectors. A detailed description of the SOM algorithm was given in Kohonen [17] and Park et al. [1].

The data were proportionally scaled between 0 and 1 in the range of the minimum and maximum values. After training the SOM, the cluster boundaries on the trained maps were further determined by a hierarchical cluster analysis with a Ward linkage algorithm using the Euclidean distance measure. Multiresponse permutation procedure (MRPP) was conducted to evaluate the significance of the clusters [18]. The MRPP is a nonparametric procedure for testing the hypothesis of no difference between groups defined by the SOM, and was performed using PC-ORD Ver. 4.25 [19].

\section{Results}

\subsection{Effects of toxic chemical treatments}

Movement patterns of medakas were analyzed at 3 different lengths: 3-, 6-, $12-$ point segments. Firstly, we analyzed the differences in movement parameters with 3-point segments, and then, the movement patterns were characterized with the SOM. When medaka were treated with copper, movement activity in terms of angle, speed, and movement distance per unit time $(0.25 \mathrm{sec})$ decreased significantly (paired $t$-test, $p<0.05$; Figure 4). Similar patterns were also observed with 6- and 12-point segments, although they are not presented in the figure. 

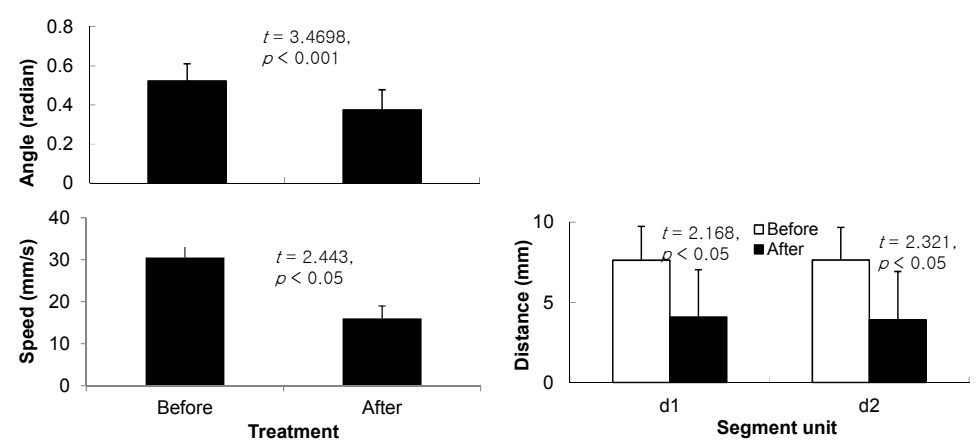

Figure 4: Differences in movement parameters of medaka responding to copper treatment.

\subsection{Classification of movement patterns}

Movement patterns with behavioural parameters (speed, angel, and distance) were classified based on the SOM learning process. Firstly, we used the data from the 3-point segments for the SOM analysis. Pooled data before and after treatment were used in the classification of movement patterns in the SOM, and the SOM results showed that their occurrence patterns were clearly different after copper treatment (Figure 5). The SOM output units were classified into 5 clusters (1-6) based on U-matrix and the dendrogram of the hierarchical cluster analysis. The classification revealed the changes in behavioral patterns caused by chemical treatment. Cluster 1 showed low values for speed, angle, and movement distances $\mathrm{d} 1$ and $\mathrm{d} 2$, indicating that movement patterns were affected

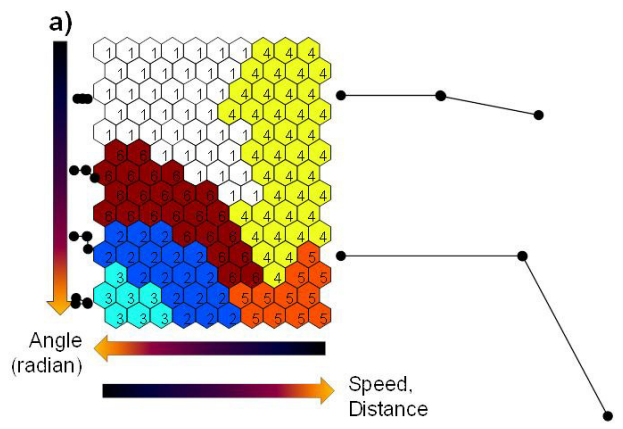

b)

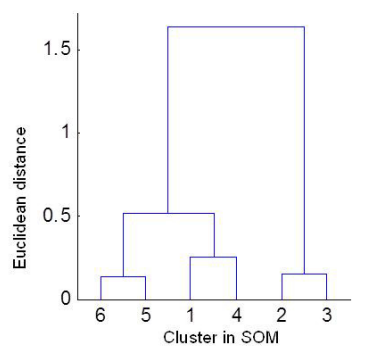

Figure 5: Classification of movement patterns in SOM based on the differences of movement parameters. b) A dendrogram of hierarchical cluster analysis with the Ward linkage method using Euclidean distance. Lines with 3 points indicate typical movement patterns in each cluster. 
by chemical treatment (Figure 6). Clusters 4 and 5 displayed high speed and long movement distances $\mathrm{d} 1$ and $\mathrm{d} 2$, representing movement patterns when untreated by copper. Clusters 2 and 3 showed a high value for angle and low values for speed and movement distance.
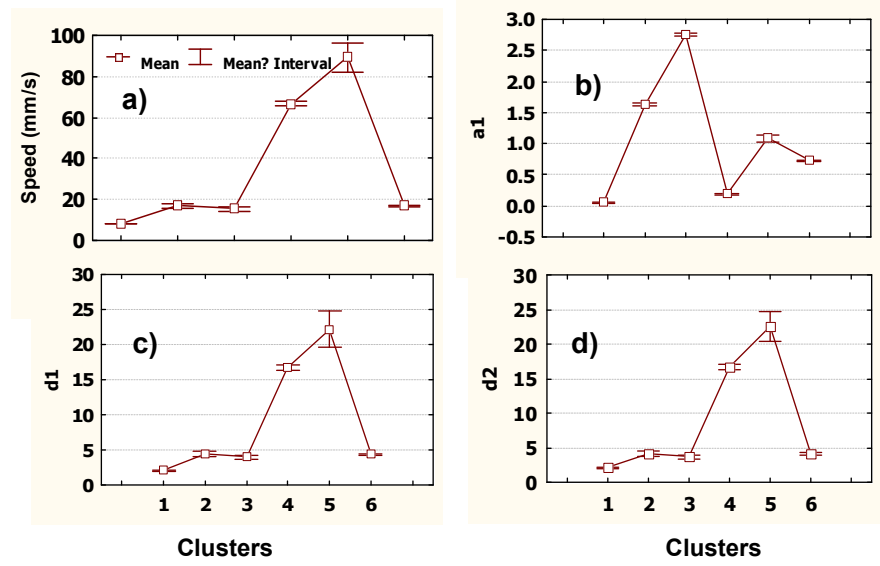

Figure 6: Differences of movement parameters measured with 3 point segments at different clusters defined in the SOM. a) speed, b) angle, c) movement distance between points 1 and 2, and d) movement distance between points 2 and 3 in 3-point segments.

\subsection{Effects of segment sizes on discrimination rate}

Based on the SOM classification, movement patterns were discriminated according to chemical treatment at different numbers of segments $(3,6$, and 12 ; $\left.\mathrm{y}=-1.3888 \mathrm{x}+64.915, R^{2}=0.9901\right)$. Correct discrimination $(\%)$ was decreased with increasing number of segmentations (Figure 7). At 3-point segmentation, correct discrimination was $61.2 \%$, while it was $48.5 \%$ at 12 -point segmentation. Our results showed that 3-point segmentation is more efficient to characterize the effects of copper on medaka, although overall discrimination rates were relatively low. This indicates that the 3 behavioural parameters used in this study (speed, angle, and distance) were not sufficient to identify the effects of copper treatment on medaka. It is clear that the 3-point segments of movement track are better than a larger number of segments. It saves computational time and cost, which is important for real-time early warning systems. However, it is necessary to investigate how the discrimination efficiency can be improved with the combination of other parameters. 


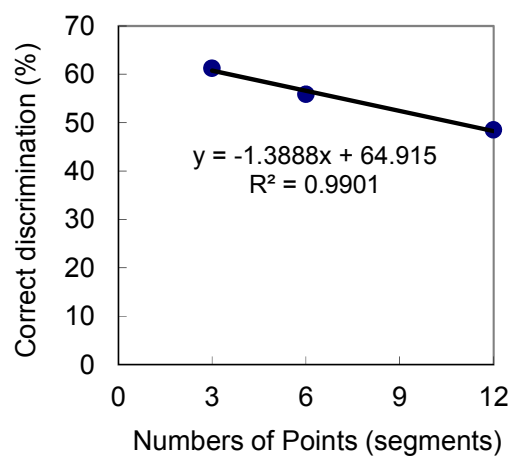

Figure 7: Changes of discrimination power for the response of medaka to copper treatment.

\section{Conclusions}

We observed the movement patterns of medaka (Oryzias latipes) in response to low concentrations $(1 \mathrm{mg} / \mathrm{L})$ of copper ion using the automatic detection system of animal movement behaviour, and the movement patterns with behavioural parameters were characterized through an unsupervised learning algorithm with SOM. Medaka movement activity after copper treatment was decreased. Changes in movement tracks caused by copper treatment were discriminated with segmentation using SOM, and 3-point segments showed higher discrimination efficiency than did 6- and 12-point segments. Further studies are required to improve discrimination rates. Finally, in situ bio-monitoring could be possible utilizing computational methods with simple movement parameters and SOM.

\section{Acknowledgement}

This study was supported by a National Research Foundation of Korea (NRF) grant funded by the Korean government (MEST) (No. 2010-0027360).

\section{References}

[1] Park, Y.-S., Chung, N.-I., Choi, K.-H., Cha, E.Y., Lee, S.-K., and Chon, T.S., Computational characterization of behavioral response of medaka (Oryzias latipes) treated with diazinon. Aquatic Toxicology, 71(3), pp. 215228, 2005.

[2] Ji, C.W., Kim, H., Chon, T.-S., and Park Y.-S., Computational analysis of movement behaviors of medaka (Oryzias latipes) in response to chemical and thermal stressors. Journal of the Korean Physical Society 60(4), pp. 570-575, 2012. 
[3] Kwak, I.-S., Chon, T.-S., Kang, H.-M., Chung, N.-I., Kim, J.-S., Koh, S.C., Lee, S.-K., and Kim, Y.-S., Pattern recognition of the movement tracks of medaka (Oryzias latipes) in response to sub-lethal treatments of an insecticide by using artificial neural networks. Environmental Pollution 120, pp. 671-681, 2002.

[4] Untersteiner, H, Kahapka, J., Kaiser, H., Behavioural response of the cladoceran Daphnia magna STRAUS to sublethal copper stress - validation by image analysis. Aquat. Toxicol. 65, pp. 435-442, 2003.

[5] Alt, W. and Hoffman, G., (Eds.) Biological Motion. Springer, Berlin, 1990.

[6] Scharstein, H., Paths of carabid beetles walking in the absence of orienting stimuli and the time structure of their motor output. In: Alt, W., Hoffmann, G., (Eds.), Biological Motion. Springer, Berlin, pp. 269-277, 1990.

[7] Johnson, A.R., Milne, B.T., and Weins, J.A., Diffusion in fractal landscapes: simulations and experimental studies of tenebrionid beetle movements. Ecology 73, pp. 1968-1983, 1992.

[8] Wiens, J.A., Crist, T.O., With, K.A. and Milne, B.T., Fractal patterns of insect movement in microlandscape mosaics. Ecology 76, pp. 663-666, 1995.

[9] Alados, C.L., Escos, J.M., and Emlen, J.M., Fractal structure of sequential behaviour patterns: an indicator of stress. Anim. Behav. 51, pp. 437-443, 1996.

[10] Ji, C.W., Lee, S.H., Kwak, I.-S., Cha, E.Y., Lee, S.-K. and Chon, T.-S., Computational analysis of movement behaviors of medaka (Oryzias latipes) after the treatments of copper by using fractal dimension and artificial neural networks. In: eds A.G. Kungolos, C.A. Brebbia, C.P. Samaras and V. Popov, Environmental Toxicology. WIT Press: Southampton and Mykonos, pp. 93-107, 2006.

[11] Liu, Y., Chon, T.-S., Lee, S.H., Hidden Markov Model and Self-organizing Map Applied to Exploration of Movement Behaviors of Daphnia magna (Cladocera: Daphniidae). J Korean Phys Soc, 56, pp. 1003-1010, 2010.

[12] Liu, Y., Lee, S.H., Chon, T.-S., 2010. Analysis of behavioral changes of zebrafish (Danio rerio) in response to formaldehyde using Self-organizing map and a hidden Markov model. Ecol Model, 222, pp. 2191-2201, 2011.

[13] Liu, Y., Chon, T.-S., Baek, H.K., Do, Y. Choi, J.H., Chung, Y.D., Permutation entropy applied to movement behaviors of Drosophila melanogaster. Mod Phys Lett B, 25, pp. 1133-1142, 2011.

[14] Wilt, F.H. , Wessells, N.K., (Eds.), Methods in Developmental Biology. T.Y. Crowell Co., New York, 1967.

[15] Us Environmental Protection Agency. 1984. Ambient Aquatic Life Water Quality for Copper, http://www.epa.gov/ost/pc/ambientwqc/ copper1984.pdf.

[16] Ji, C.W., Lee, S.H., Choi, K.-H., Kwak, I.-S., Lee, S.G., Cha, E.Y., Lee, S.K. and Chon, T.-S., Monitoring of movement behaviors of chironomid larvae after exposure to diazinon by using fractal dimension and SelfOrganizing Map. Ecological Dynamics, 2, pp. 1-12, 2008.

[17] Kohonen, T., Self-Organizing Maps. Springer, Berlin, 2001. 
146 Environmental Impact

[18] Mielke, E.W., Berry, K.J., and Johnson, E.S., Multiresponse permutation procedures for a priori classifications. Commun. Stat. Theor. M. 5, pp. 1409-1424, 1976.

[19] McCune, B., Mefford, M.J., PC-ORD. Multivariate Analysis of Ecological Data. Version 4.41.MjM Software, Gleneden Beach, Oregon, 1999. 